\title{
A case of axial spondyloarthritis with Paget's disease: A rare coexistence
}

\author{
Emre Tekgöz ${ }^{1}$, Muhammet Çınar ${ }^{1}$, Hatice Tuba Sanal ${ }^{2}$, Sedat Yılmaz ${ }^{1}$ \\ (1) University of Health Sciences, Gulhane Faculty of Medicine, Department of Internal Medicine, Division of \\ Rheumatology, Ankara, Turkey \\ (2) University of Health Sciences, Gulhane Faculty of Medicine, Department of Radiology, Ankara, Turkey
}

Date submitted:

Nov 05, 2018

Date accepted:

Dec 29, 2018

Online publication date:

June 15, 2019

\section{Corresponding Author: \\ Emre Tekgöz}

University of Health Sciences,

Gulhane Faculty of Medicine,

Department of Internal Medicine,

Division of Rheumatology,

Ankara, Turkey

dr.emretekgoz@hotmail.com

Keywords: Alkaline phosphatase, Paget's disease, SpondyloArthritis.

\begin{abstract}
Paget's disease (PD) is one of the most common bone disease which bone destruction and construction cycle is abnormally accelerated. Disease develops under the influence of environmental factors at genetic background. Pain is the most common complaint. Especially the waist and hip region pain might be confused with inflammatory spondyloArthritis. High serum alkaline phosphatase value is important in diagnosis. Direct $X$ - rays, computed tomography, magnetic resonance imaging and bone scintigraphy are the most important imaging methods for suspected patients. In rare cases these two diseases can be seen together and distinguish these diseases might be difficult. In our case, referring to PD accompanying an axial spondyloArthritis patient who complained of low back pain is presented.
\end{abstract}

\section{Introduction}

Paget's disease (PD) is one of the most common diseases of the bone. The most important mechanism in the pathogenesis is the formation of new bone with poor quality as a result of irregularities in bone formation and destruction $(1,2)$. The elevation of serum alkaline phosphatase levels is the most important laboratory abnormality. In the suspected patients, specific changes observed on direct radiographs, computed tomography (CT), magnetic resonance (MR) imaging and bone scintigraphy are very important in diagnosis. The primary treatment that prevents bone abnormal formation in diagnosed patients is bisphosphonates. Ankylosing spondylitis (AS) is a chronic, systemic, inflammatory disease that primarily causes inflammation in the sacroiliac joints and spine. The most important complaint of patients with AS is the pain of the hip and lower back. The feature of the pain has an important place in the distinction between these two clinical entities. The distinction of AS from PD, where involvement of the hip bones is rarely observed, is very important in terms of clinical, treatment and follow-up. However, it should be kept in the mind that rarely these two diseases can coexist. In our case, referring to PD accompanying an axial
spondyloArthritis (axSpA) patient who complained of low back pain is presented.

\section{Case}

A 36-year-old male patient who was followed up in remission under etanercept therapy with axSpA diagnosis was admitted to our out-patient clinic because of mechanical low back pain for approximately continued one month. In the examination, sacroiliac compression tests were negative and no arthritis was detected in peripheral joints. In laboratory values, acute phase response was normal and serum alkaline phosphatase level was found to be as high as $750 \mathrm{U} / \mathrm{L}$ (normal level 25-100 U/L). In plain radiography, there was irregularity and sclerosis on the right sacroiliac joint and sclerosis on the left (Figure 1). Although the recent low back pain of the patient was not typically inflammatory, the sacroiliac joint MR imaging was performed to exclude active sacroiliitis. There was no bone marrow edema at sacroiliac joints. Instead, a heterogeneous area with thick trabeculae on T1-weighted images, hyperintense on fluid sensitive images with enhancement after intravenous contrast media administration was detected on the right iliac wing next to 
the sacroiliac joint (Figure 2). Bone scintigraphy was obtained because of the elevated serum alkaline phosphatase and MR abnormalities. In this respect, the increased activity was found in the lesion when compared to its symmetry of the right hemi pelvis was considered as compatible with PD. The patient diagnosed with PD and administered zoledronic acid intravenously. With treatment, complaints of the patient were relieved and serum alkaline phosphatase levels were decreased. During this period, etanercept was continued for the diagnosis of axSpA.

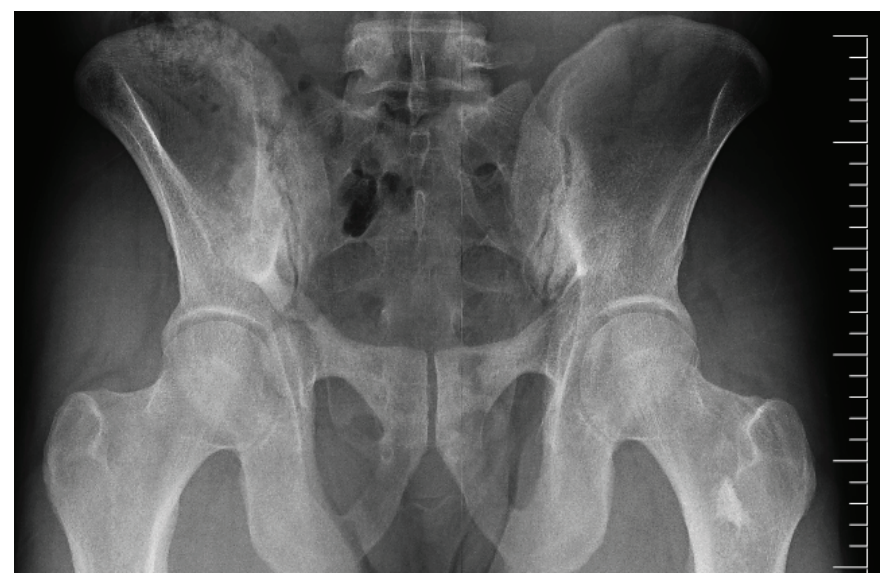

Figure 1. $\mathrm{X}$ ray of both sacroiliac joints showing irregularity and sclerosis on the right iliac surface and only slight sclerosis on the left side.

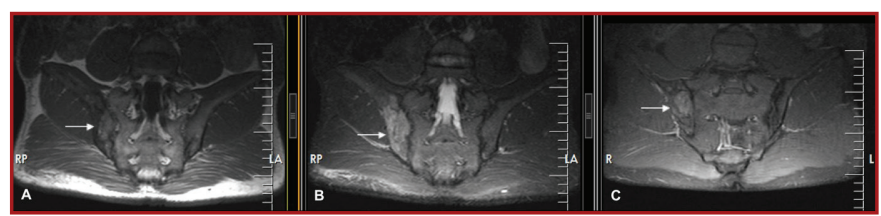

Figure 2. T1-weighted (a) FST2-weighted (b) and post contrast FST1 (c) weighted MR images of both sacroiliac joints acquired in coronal plane. Please note the pagetic area on the right iliac bone next to the sacroiliac joint with its coarse thick trabeculae observed with hyperintensity on FST2 and enhancing on postcontrast images (arrow).

\section{Discussion}

$\mathrm{PD}$ is the second most common disease of the bone following osteoporosis. Although the etiology is not fully understood, autosomal dominant transitions are observed and mutations in the sequestosome-1 (SQSTM1) gene are most frequently detected (1). In pathogenesis, there is an abnormal increase in bone destruction and construction in every stage. However, the new bone that is formed is not as robust as the old one. The most common symptoms are pain, neurological complaints, skeletal deformities, pathological fractures and deafness, although the disease is usually asymptomatic (2). The important cause of pain is the functional limitation due to degenerative changes, not the pagetic bone lesion (3). Mostly the disease is localized in a single bone. In clinical practice, serum alkaline phosphatase level is the most commonly used biomarker in diagnosis and follow-up of disease activity, indicating osteoblast activity. Measurements showing bone formation (bone specific alkaline phosphatase, osteocalcin, procollagen type 1 peptide) and bone resorption (pyridino lines, hydroxyproline, cross linked collagens) do not show any advantage over serum alkaline phosphatase levels. Therefore it is advantageous that the serum alkaline phosphatase values are following, easily accessible and cheap. Clinical, laboratory and radiological findings are sufficient in the diagnosis of the disease. If there is any doubt in radiological findings, biopsy from pagetic bone is recommended (2). Bisphosphonates, which inhibits osteoclast activity, are the most preferred drugs in the treatment of symptomatic patients. Currently, intravenous zolendronic acid treatment seems optimal in bisphosphonates compared with others (4). Although PD is frequently seen in the skull and long bones, it can also be seen in the pelvic bones. The involvement of the sacroiliac joints may lead to conceptual confusion with $\operatorname{axSpA}$, which is very specific in terms of involvement of the sacroiliac joint. Axial SpA is a chronic inflammatory rheumatic disease that primarily causes inflammation in the sacroiliac joints and spine. Although the findings of sacroiliac radiograph provide convenience for distinguishing these two diseases, these clinical entities are often confused with each other. PD lesions typically do not extend to healthy joints. Very importantly, we should not forget that the disease does not cross the joint lines and the pelvic region settled pagets' disease seperates the sacrum and the hemi-pelvis (2).

\section{Conclusion}

In rheumatology practice, the character of the low back pain in a patient is very important in terms of diagnosis. In this respect, it is important to assess the patients' history carefully and ask about the nature of the pain the patient suffering from. In this case, PD diagnosis was made based on the mechanical low back pain, specific images seen on the MRI and scintigraphy. There was also a high level of serum alkaline phosphatase accompanying the present findings. At the same time, the patient met the Assessment of SpondyloArthritis international Society axSpA criteria and was in remission with etanercept. In addition, when the findings of the patient diagnosed with spondyloArthritis were re-evaluated, it was determined that the level of serum alkaline phosphatase was normal and there were no findings in the right iliac wing of sacroiliac MR image. In this present case, we have referred to the rare association of axS$\mathrm{pA}$ and PD and have presented our clinical experience in the diagnosis and follow-up.

Acknowledgment: ET, M.D. Conception, Design, Data Collection, Writer MÇ, M.D. Supervision, Materials, Analysis and Interpretation, Writer HTS, Supervision, Writer, Literature Review, Critical Review SY, M.D., Supervision, Writer, Literature Review, Critical Review

Conflict of Interest: The authors declared they do not have anything to disclose regarding conflict of interest with respect to this manuscript.

\section{References}

1. Laurin N, Brown JP, Morissette J, Raymond V. Recurrent mutation of the gene encoding sequestosome 1 (SQSTM1/ p62) in Paget disease of bone. Am J Hum Genet. 2002;70(6):1582-1588.

2. Seton M. Paget disease of bone:diagnosis and drug therapy. Cleveland Clinic journal of medicine. 2013;80(7):452-462.

3. Seton M. Diagnosis, complications and treatment of $\mathrm{Pa}-$ get's disease of bone. Aging Health. 2009; 5(4):497-508.

4. Reid IR, Miller P, Lyles K, Fraser W, Brown JP, Saidi Y, et al. Comparison of a single infusion of zoledronic acid with risedronate for Paget's disease. New England Journal of Medicine. 2005;353(9):898-908. 